\title{
Evaluation of Helping Babies Breathe Quality Improvement Cycle (HBB-QIC) on retention of neonatal resuscitation skills six months after training in Nepal
}

Ashish $\mathrm{KC}^{1,2^{*}}$, Johan Wrammert ${ }^{1}$, Viktoria Nelin ${ }^{1}$, Robert B. Clark ${ }^{3}$, Uwe Ewald ${ }^{1}$, Stefan Peterson ${ }^{1,4,5,6}$ and Mats Målqvist ${ }^{1}$

\begin{abstract}
Background: Each year 700,000 infants die due to intrapartum-related complications. Implementation of Helping Babies Breathe (HBB)-a simplified neonatal resuscitation protocol in low-resource clinical settings has shown to reduce intrapartum stillbirths and first-day neonatal mortality. However, there is a lack of evidence on the effect of different HBB implementation strategies to improve and sustain the clinical competency of health workers on bag-and-mask ventilation. This study was conducted to evaluate the impact of multi-faceted implementation strategy for $\mathrm{HBB}$, as a quality improvement cycle (HBB-QIC), on the retention of neonatal resuscitation skills in a tertiary hospital of Nepal.

Methods: A time-series design was applied. The multi-faceted intervention for HBB-QIC included training, daily bag-and-mask skill checks, preparation for resuscitation before every birth, self-evaluation and peer review on neonatal resuscitation skills, and weekly review meetings. Knowledge and skills were assessed through questionnaires, skill checklists, and Objective Structured Clinical Examinations (OSCE) before implementation of the HBB-QIC, immediately after HBB training, and again at 6 months. Means were compared using paired t-tests, and associations between skill retention and HBB-QIC components were analyzed using logistic regression analysis.

Results: One hundred thirty seven health workers were enrolled in the study. Knowledge scores were higher immediately following the HBB training, $16.4 \pm 1.4$ compared to $12.8 \pm 1.6$ before (out of 17), and the knowledge was retained 6 months after the training (16.5 \pm 1.1$)$. Bag-and-mask skills improved immediately after the training and were retained 6 months after the training. The retention of bag-and-mask skills was associated with daily bag-and-mask skill checks, preparation for resuscitation before every birth, use of a self-evaluation checklist, and attendance at weekly review meetings. The implementation strategies with the highest association to skill retention were daily bag-and-mask skill checks (RR-5.1, 95\% Cl 1.9-13.5) and use of self-evaluation checklists after every delivery (RR-3.8, 95\% Cl 1.4-9.7).

(Continued on next page)
\end{abstract}

\footnotetext{
* Correspondence: aaashis7@yahoo.com; http://www.kbh.uu.se/imch

${ }^{1}$ International Maternal and Child Health, Department of Women's and

Children's Health, Uppsala University, Uppsala, Sweden

United Nation's Children's Fund (UNICEF), Nepal Country Officer, UN House,

Lalitpur, Nepal

Full list of author information is available at the end of the article
} 
(Continued from previous page)

Conclusions: Health workers who practiced bag-and-mask skills, prepared for resuscitation before every birth, used self-evaluation checklists, and attended weekly review meetings were more likely to retain their neonatal resuscitation skills. Further studies are required to evaluate HBB-QIC in primary care settings, where the number of deliveries is gradually increasing.

Trial registration: ISRCTN97846009. Date of Registration- 15 August 2012.

Keywords: Neonatal resuscitation, Helping babies breathe, Retention of skills, Multi-faceted implementation strategy, Quality improvement cycle, Nepal

\section{Background}

Globally, 2.9 million neonates die every year, of which 700,000 die due to intrapartum-related complications [1]. The first minute after a baby is born-the "Golden minute ${ }^{\text {Tum }}$ - is the crucial window for the initiation of neonatal resuscitation among the 10 million nonbreathing babies born annually [2]. Neonatal resuscitation competency among health workers is therefore critical in every delivery room to ensure the safety and health of newborn infants. Resuscitation training in facilities has been shown to reduce the number of intrapartum-related neonatal deaths by $30 \%$ [3]. Although health workers are the key personnel in the provision of neonatal resuscitation, it has been revealed that in low-resource settings staff usually have inadequate training and knowledge, and that additional formal guidance and support are needed $[4,5]$.

Nepal has a neonatal mortality rate of 23 per thousand live births and intrapartum-related complications account for $23 \%$ of neonatal deaths [1, 6]. In Nepal, more than half of all deliveries take place at health institutions, but the quality of resuscitation provided at the time of birth has been found to be inadequate [7]. Identifying the implementation strategies that could improve and sustain neonatal resuscitation competency will be key to improve the quality of resuscitation care provided.

There are a number of intervention strategies that have been identified to improve the performance and competencies of health workers for a number of other clinical guidelines [8]. However, strategies to improve and sustain competency on neonatal resuscitation have not been evaluated.

Helping Babies Breathe ${ }^{-}$(HBB), developed by the American Academy of Pediatrics, is designed to train birth attendants in developing countries on the essential skills of neonatal resuscitation [9]. It is based on evidence from a neonatal evaluation study done by ILCOR (International Liaison Committee on Resuscitation), and recognizes that in many countries only one birth attendant is present to provide care to both the mother and the newborn $[10,11]$.

The educational material of HBB consists of a welltested pictorial representation of the resuscitation protocol, learner workbooks, facilitator flip charts, neonatal simulators and the required equipment - i.e., reusable ventilation bag-and-mask devices and bulb suction devices [9]. An evaluation of the HBB program in Tanzania showed that intrapartum-related stillbirth and early neonatal mortality was reduced by $24 \%$ and $47 \%$, respectively [12]. The Tanzanian study showed that the $\mathrm{HBB}$ program is a low-cost intervention, with the estimated cost per life saved at USD 233 and USD 4.21 per life year gained [13]. However, implementation strategies for HBB in the Tanzania study were not evaluated in relation to the retention of neonatal resuscitation competencies among health workers.

An evaluation study conducted in Ethiopia found that HBB training improved the neonatal resuscitation knowledge of health workers immediately after training and also eliminated the knowledge difference that was present prior to training among health workers [14]. A study from Rwanda revealed that neonatal resuscitation competency dropped to an unsatisfactory level 3 months after the training, indicating that training alone was not adequate to retain the health worker's neonatal resuscitation knowledge and skills [15]. The proportion of providers at a rural hospital in Tanzania who were competent in simulated routine care and neonatal resuscitation scenarios increased after HBB training, and their knowledge remained at 7 months after training; however, the improvement did not transfer into clinical competency [16]. There is thus a need to further evaluate multi-faceted interventions to promote retention of resuscitation skills, as well as to make sure that knowledge is translated into clinical practice.

We therefore conducted this study to evaluate the impact of a multi-faceted implementation strategy for HBB as a Quality Improvement Cycle (HBB-QIC), on change and retention of health workers' knowledge and skills of neonatal resuscitation.

\section{Methods}

We conducted this study in a tertiary hospital in Kathmandu, Nepal. In 2011, the hospital had around 22,000 deliveries, and an early neonatal mortality of nine per thousand live births [17]. The hospital 
provides level III obstetric and gynecological services and is publicly funded.

Labor and delivery services are provided in the admission unit, antenatal care unit, labor unit, maternal and newborn service center (MNSC), and the operation theatre. The hospital also has a system that rotates the staff of these units on a periodic basis.

This was a sub-set of a larger study conducted to evaluate the change in perinatal mortality before and after the implementation of a simplified neonatal resuscitation protocol, the HBB-QIC, in the hospital [18]. The duration of the larger study was 18 months, from July 2012 to September 2013. As part of the larger study evaluating the impact of neonatal resuscitation protocol implementation, the study received approval from the Hospital's Institutional Review Committee, the Nepal Health Research Council (Reg. No. 37/2012) and the Ethical Review Board of Uppsala University (dnr 2012/ 267). The study was registered as clinical trial, ISRCTN 97846009 [18].

All 137 nurses working in the admission unit, antenatal care unit, labor unit, MNSC, operation theatre and postnatal ward were included in the study. Informed written consent was obtained from each health worker participating in the study.

\section{Study design}

This was a time-series design where a series of periodic measurements were completed to evaluate our multifaceted implementation strategy for the HBB-QIC, at three different time points (Table 1).
The knowledge and skills of health workers were assessed using the standard tools included in the HBB package, which were validated and used in other settings where $\mathrm{HBB}$ knowledge and skill evaluations have been conducted [9]. A questionnaire with 17 multiple-choice questions was used to assess the knowledge of health workers on the HBB protocol. The following tools were used to evaluate the skills of health workers in using the HBB protocol: a 7-step checklist for bag-and-mask skill checks, a 5-step checklist for preparation for resuscitation before every birth skill checks, a 13-step checklist with simulation for the first Objective Structured Clinical Examination (OSCE A), and a 18-step checklist with simulation for OSCE B. Two OSCEs tested healthcare worker's ability to prepare for, assess, and act in a scenario of routine newborn care (OSCE A) and a scenario requiring bag-and-mask ventilation (OSCE B) [9]. Successful completion of each OSCE required correct overall performance $(\geq 80 \%)$, as well as the completion of key assessments and interventions such as "recognizes baby not breathing/crying" and "provides bag-and-mask ventilation" [9]. Assessment using these tools was completed before the HBB training was provided, immediately after the training, and again at 6 months after completion of the training (Table 1).

Information on each health worker's daily routines, including daily bag-and-mask skill checks, preparation for resuscitation before every birth, self-evaluation checklist usage, completion of the peer evaluation process, and attendance at the weekly review meetings was assessed using a direct observation checklist (Table 1).

Table 1 Evaluation of HBB QIC

\begin{tabular}{|c|c|c|c|}
\hline & Thematic area & Tools & Evaluation design \\
\hline \multirow[t]{5}{*}{ Knowledge and skill competency } & Knowledge assessment & 17 multiple choice questions & $\begin{array}{l}\text { Before training, immediately after } \\
\text { training and } 6 \text { months after training }\end{array}$ \\
\hline & Bag-and-mask skill & 7 step skill observation checklist & $\begin{array}{l}\text { Before training, immediately after } \\
\text { training and } 6 \text { months after training }\end{array}$ \\
\hline & Preparation at birth & 5 step skill observation checklist & $\begin{array}{l}\text { Before training, immediately after } \\
\text { training and } 6 \text { months after training }\end{array}$ \\
\hline & OSCE A & 13 step observation checklist & $\begin{array}{l}\text { Before training, immediately after } \\
\text { training and } 6 \text { months after training }\end{array}$ \\
\hline & OSCE B & 18 step observation checklist & $\begin{array}{l}\text { Before training, immediately after } \\
\text { training and } 6 \text { months after training }\end{array}$ \\
\hline \multirow[t]{5}{*}{ Clinical practice } & Daily skill check & 7 step skill observation checklist & $\begin{array}{l}\text { Observation on a daily basis using a } \\
\text { checklist }\end{array}$ \\
\hline & $\begin{array}{l}\text { Preparation for resuscitation } \\
\text { before every birth }\end{array}$ & 5 step skill observation checklist & $\begin{array}{l}\text { Observation on a daily basis using a } \\
\text { checklist }\end{array}$ \\
\hline & Self-evaluation checklist & 21 step checklist & $\begin{array}{l}\text { Observation on a daily basis using a } \\
\text { checklist }\end{array}$ \\
\hline & Peer evaluation process & HBB schematic protocol & $\begin{array}{l}\text { Observation on a daily basis using a } \\
\text { checklist }\end{array}$ \\
\hline & Weekly review meetings & Notes of the meeting & $\begin{array}{l}\text { Observation on a daily basis using a } \\
\text { checklist }\end{array}$ \\
\hline
\end{tabular}




\section{HBB QIC intervention}

The HBB-QIC was implemented from January until September 2013. The HBB-QIC included multi-faceted implementation strategy including: HBB training, daily bag-and-mask skill checks, preparation for resuscitation before every birth, self-evaluation and peer review on neonatal resuscitation skills and weekly review meetings (Table 2).

First, a two-day training package on the HBB-QIC was provided to all hospital staff working in the three delivery units. There were 6 trainers who trained all the staff in each of the delivery units in a cascade manner. During the study period, there was no rotation of staff among the delivery units. Following the training, bag-and-mask kits and penguin (bulb) suctions ${ }^{\mathrm{Tt}}$ were provided to each delivery unit. Additionally, HBB mannequins were placed at the entry of each delivery unit for daily skill checks; self-evaluation checklists were also attached to each clinical record form; and HBB schematic posters were placed in front of each resuscitation table for peer review. Weekly review meetings were conducted by each unit in-charge to discuss progress on the implementation of HBB-QIC standards.

\section{Data management}

In order to complete sampling and data collection, an independent surveillance team was formed. The team consisted of eight female surveillance officers with an academic background in nurse-midwifery and sufficient experience in clinical research. A research manager supervised the activities of this surveillance team. The surveillance team was trained to collect information using an observation checklist. Surveillance officers who conducted the HBB trainings collected information from the study participants, including demographic characteristics, academic qualifications and clinical experience.

After the surveillance officers collected this information, it was given to the data entry officer for dataset creation and management. The information was reviewed for completeness and then entered into an electronic database that was developed in the Census and Survey Processing System (CS Pro) software (US Census Bureau, ICF Macro). The data was then cleaned and transferred into the Statistical Package for the Social Sciences (SPSS) software (IBM Corporation) for data analysis.

\section{Data analysis}

The background characteristics of the health workers that were analyzed included age, professional experience, number of deliveries attended per month, number of neonatal resuscitations conducted per month and academic qualifications. The mean $\pm \mathrm{SD}$ and median (IQR) for age of the health worker were calculated; mean and median years of professional experience in midwifery were calculated; the mean and median number of deliveries and resuscitations attended per month were also calculated. Academic qualifications were categorized into two groups, those who had completed an intermediate level of education in nursing or who were axillary nurse midwives compared to those who had completed a bachelor in nursing or higher.

Table 2 Description of the multi-faceted implementation strategy for Helping Babies Breathe (HBB) Quality Improvement Cycle (QIC)

\begin{tabular}{lll}
\hline Component & Activity & Facilitators and participants \\
\hline HBB training & Two-day training: First day on HBB knowledge and skills as per & Facilitators: HBB trainers \\
& standard package and second day on components of HBB QIC & Participants: Staff of the delivery units
\end{tabular}
Fir standards, training of trainers on how to conduct weekly review meeting, how to fill self-evaluation checklists and conduct peer evaluations.

Setting up HBB QIC standards

At each unit: Development of QIC goals and objectives, development of a place for daily bag-and-mask skill checks, QIC weekly review meetings, use of self-evaluation checklists and peer reviews after each resuscitation.

QIC Weekly review meeting

Daily bag-and-mask skill check

Self-evaluation checklist after each delivery

Peer review after each resuscitation
At each unit, the unit in-charge facilitates the weekly review meetings on the progress of implementation of HBB QIC standards.

At each unit, each staff does a bag-and-mask skill check on a mannequin before starting duty.

A self-evaluation checklist, which consists of a list of steps for immediate newborn care and neonatal resuscitation as per HBB protocol with checkboxes. After completing care of each newborn the nurse midwife will fill up the self-evaluation checklist based on the steps completed as per the HBB protocol.

A mounted poster with the steps of the HBB protocol will be attached at each resuscitation table, so that peers can review with the colleague completing resuscitation on whether the steps were followed.
Facilitators: Study team

Participants: Staff of the delivery units

Facilitators: HBB trainers

Participants: Staff of the delivery units

Facilitators: Unit in-charge

Participants: Staff of the delivery units

Facilitators: Unit in-charge

Participants: Staff of the delivery units

Facilitators: Unit in-charge

Participants: Staff of the delivery units 
The mean numeric scores obtained on the knowledge questionnaire, preparation for resuscitation before every birth checklist, bag-and-mask skill check, and OSCEs A and $\mathrm{B}$ at baseline, immediately after the training, and at 6 months after the training were compared using paired $\mathrm{t}$-tests. The proportion of health workers who scored $\geq 80 \%$ on the bag-and-mask skill checks before the training, immediately after the training, and at 6 months after the training was compared using paired t-tests.

The retention of bag-and-mask skills was calculated based on the change in bag-and-mask skill scores immediately after the training compared to 6 months after the training. The health workers who scored the same or better at 6 months after the training, as compared to immediately after training, were categorized as having retained their skills.

We then analyzed the association $(p<0.01)$ between retention of skills and health worker's completion of HBB-QIC components - i.e., daily bag-and-mask skill checks, preparation for resuscitation before every birth, use of self-evaluation checklists, peer evaluation following each resuscitation occurrence, attendance at weekly meetings - as well as with the completion of at least one neonatal resuscitation per month using the Fischer's exact test.

We also conducted logistic regression analysis to assess the level of association between the completion of each implementation strategy included in the HBB-QIC and the retention of bag-and-mask skills at 6 months.

Missing data that occurred randomly was adjusted for using the multiple imputation method [19].

\section{Results}

All 137 health workers included in this study participated in knowledge and skill evaluation before the HBB training, immediately after training and at 6 months after the training. The mean age of the health workers was $31.8 \pm 10.2$ years and the median age was 27.0 (IQR 24.0-39.0) years. Among the health workers, the mean number of professional years of experience was $10.5 \pm$ 3.3 years and the median was 9 (IQR 6.0-15.0) years. The mean number of deliveries attended by each health worker was $8.0 \pm 5.5$ and the median was 7.0 (5.0-9.0). The mean number of resuscitations conducted by each health worker per month was $3.0 \pm 1.5$ and median was 3.0 (2.0-4.0). More than half, $65 \%$, of health workers had intermediate level nursing qualifications or were axillary nurse-midwives, and the remaining health workers had bachelor degrees in nursing or higher (Table 3).

There was a significant change $(<0.001)$ in the mean knowledge score after the HBB training, $16.4 \pm 1.4$ compared to $12.8 \pm 1.6$, and the knowledge was retained 6 months after the training $(16.5 \pm 1.1)(p=0.6)$. There was a significant change $(<0.001)$ from baseline in the
Table 3 Background characteristics of the Health workers-nurse

\begin{tabular}{lll}
\hline Background Characteristics & Mean \pm SD & Median (IQR) \\
Age in complete years & $31.8 \pm 10.2$ & 27.0 (24.0-39.0) \\
$\begin{array}{l}\text { Professional experience in } \\
\text { midwifery in complete years }\end{array}$ & $10.5 \pm 3.3$ & 9.0 (6.0-15.0) \\
$\begin{array}{l}\text { Number of deliveries attended } \\
\text { per month }\end{array}$ & $8.0 \pm 5.5$ & 7.0 (5.0-9.0) \\
$\begin{array}{l}\text { Number of resuscitation } \\
\text { Academic Qualifications }\end{array}$ & $3.0 \pm 1.5$ & 3.0 (2.0-4.0) \\
$\begin{array}{l}\text { Axillary nurse midwives/ } \\
\text { intermediate in nursing }\end{array}$ & Frequency & \\
Bachelor in nursing & $90(65.7)$ & \\
\hline
\end{tabular}

mean skills scores for preparation for resuscitation before every birth, bag-and-mask skills, OSCE A, and OSCE B after the training, which was retained 6 months after the training (Table 4).

There were no health workers who were competent in bag-and-mask skills before the training; 93\% of health workers were competent immediately after the training $(p<0.001)$; and $99 \%$ of the health workers were competent 6 months after the training $(p=0.8)$ (Fig. 1).

More than half of the health workers practiced their bag-and-mask skills daily, $61 \%$ of them prepared for resuscitation before every birth, $82 \%$ of them used selfevaluation checklists after every birth, $43 \%$ of them completed peer evaluation after each resuscitation and $85 \%$ of them attended weekly review meetings (Table 5).

There was a positive association $(p<0.01)$ between retention of bag-and-mask skills and health worker's completion of daily bag-and-mask skill checks, preparation for resuscitation before every birth, use of self-evaluation checklists and attendance at weekly review meetings (Table 6).

The regression analysis showed that health workers who conducted daily bag-and-mask skill checks were five times more likely to retain neonatal resuscitation skills than those who did not (RR-5.1, 95\% CI 1.9-13.5). Health workers who prepared for resuscitation before every birth had a two-fold increased likelihood of retaining the skills than those who did not (RR-2.4, 95\% CI 1.0-5.6). Health workers who used self-evaluation checklists after each delivery were four times more likely to retain their skills than those who did not (RR-3.8, 95\% CI 1.4-9.7). Health workers that attended weekly meetings had a 2.6 times increased likelihood of retaining their skills than those who did not attend (RR-2.6, 95\% CI 1.0-7.4) (Table 7).

\section{Discussion}

We applied different implementation strategies as part of a HBB-QIC to improve the knowledge and 
Table 4 Changes in knowledge and completion of preparation at birth, bag-and-mask skill checks, and OSCEs before, immediately after and 6 months after HBB training

\begin{tabular}{lllll}
\hline & Before the training $(N=137)$ & $\begin{array}{l}\text { Immediately after training } \\
(N=137)\end{array}$ & $\begin{array}{l}\text { Follow up after } 6 \text { months } \\
(N=137)\end{array}$ \\
& Mean \pm SD & Mean \pm SD & $p$-value & Mean \pm SD
\end{tabular}

competency at different time points during the intervention period. Our study showed health workers who conducted bag-and-mask skill checks daily, who were prepared for resuscitation before every birth, who used self-evaluation checklists after each delivery, and those who attended weekly review meetings were more likely to retain their neonatal resuscitation skills, even at 6 months after the completion of training.

There are, however, certain limitations to our study. First, this study had a time-series design, thus the effectiveness of each individual component within the HBBQIC cannot be evaluated, and we can only derive the association between the implementation strategy as a whole and the retention of neonatal resuscitation skills. Second, the use of self-evaluation checklists was evaluated based on whether these forms were completed correctly by the participants along with the other forms that had to be completed following every delivery. Therefore, there may be some discrepancy between actual practice and what was recorded. The final limitation was the presence of surveillance officers to observe the implementation of the HBB QIC, especially the bag-and-mask skill checks and weekly review meetings, which might have a Hawthorne effect (observer's bias) in changing health worker's behaviors. The strength of our study was that we used a

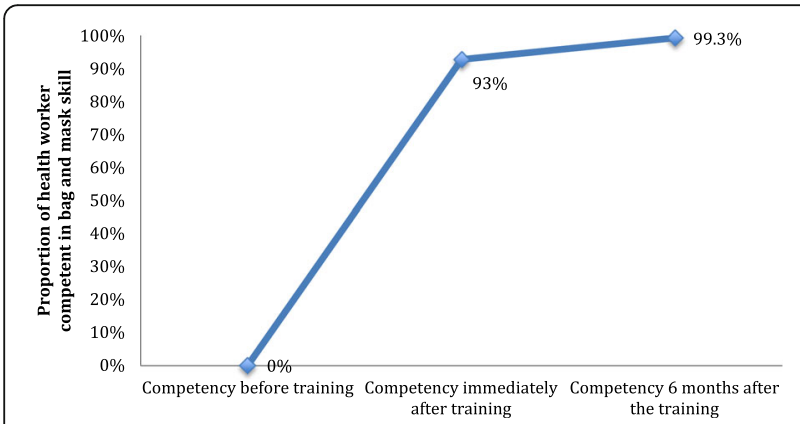

Fig. 1 Change in the proportion of the health worker who were competent $(\geq 80 \%)$ in bag and mask skill tested tool to evaluate the neonatal resuscitation knowledge and skills of the health worker [9].

Several studies in high-income and low-income countries have shown that resuscitation knowledge and skills improve immediately following training, however, these resuscitation skills tend to deteriorate over a period of time [15, 20-25]. Therefore, neonatal resuscitation training in itself is not an effective implementation strategy to retain resuscitation skills. Similar to our findings from this study, a study done in Canada has shown that the review of schematic posters on neonatal resuscitation before or after resuscitation of babies is not an effective strategy for retention of neonatal resuscitation skills [26].

Several systematic reviews have shown that the review of clinical performance among health workers on a periodic basis provides an opportunity to share the challenges faced in the clinical setting and to improve the

Table 5 Level of participation in the Helping Babies Breathe (HBB) Quality Improvement Cycle (QIC)

\begin{tabular}{lcc}
\hline & Frequency $(N=137)$ & Percent \\
\hline Frequency of bag-and-mask skill checks & 71.8 \\
Every day & 76 & 33.6 \\
2 times a week & 20 & 14.6 \\
Weekly & 84 & \\
Frequency of preparation for resuscitation before every birth & \\
At every birth & 30 & 38.3 \\
Occasionally & \\
Frequency of practicing self-evaluation & 81.8 \\
For every birth & 112 & 18.2 \\
Occasionally & 25 & \\
Frequency of practicing peer evaluation & 43.0 \\
For every resuscitation & 59 & 57.0 \\
Occasionally & 78 & \\
Attendance of weekly review meeting & 117 & \\
Yes & 20 & 14.6 \\
No & & \\
\hline
\end{tabular}


Table 6 Association between the different implementation strategies of the Helping Babies Breathe (HBB) Quality Improvement Cycle $(\mathrm{QIC})$ and the retention of bag-and-mask skills

\begin{tabular}{lllll}
\hline & & $\begin{array}{l}\text { Non-retention } \\
(N=27)\end{array}$ & $\begin{array}{l}\text { Retention } \\
(N=110)\end{array}$ & $p$-value \\
\hline $\begin{array}{l}\text { Daily bag-and-mask skill } \\
\text { checks }\end{array}$ & Yes & $6(22.2 \%)$ & $65(40.9 \%)$ & $p=0.001$ \\
No & $21(77.8 \%)$ & $45(59.1 \%)$ & \\
$\begin{array}{l}\text { Preparation for resuscitation } \\
\text { before every birth }\end{array}$ & Yes & $12(44.4 \%)$ & $72(65.5 \%)$ & $p=0.038$ \\
& No & $15(55.6 \%)$ & $38(34.5 \%)$ & \\
$\begin{array}{l}\text { Use of self-evaluation } \\
\text { checklist }\end{array}$ & Yes & $17(63.0 \%)$ & $95(86.4 \%)$ & $p=0.008$ \\
Peer evaluation after each & Yes & $12(44.4 \%)$ & $47(42.7 \%)$ & $p=0.5$ \\
resuscitation & No & $15(55.6 \%)$ & $63(57.3 \%)$ & \\
Attendance of weekly & Yes & $20(74.1 \%)$ & $97(88.2 \%)$ & $p=0.065$ \\
review meetings & No & $7(25.9 \%)$ & $13(11.8 \%)$ & \\
\hline
\end{tabular}

clinical skills and performance as a team, especially if the baseline performance on the clinical protocol is poor [27-30]. We showed that weekly review meetings among the nursing staff on the clinical performance of resuscitation helped to improve resuscitation skills to a moderate extent, as the baseline neonatal resuscitation competency was poor in this setting. Systematic reviews have also shown that a combination of implementation strategies (such as weekly review meetings, periodic simulated skill checks, checklists, and self-evaluation) is a more effective strategy to improve clinical performance as compared to the use of a singular strategy [31-36]. However, these implementation strategies were never implemented in combination to improve and increase the retention of neonatal resuscitation skills.

In the context of Nepal, the current number of neonatal deaths due to intrapartum-related complications needs to be reduced by at least three quarters in order to reach the pledged Every Newborn Action Plan target of a neonatal mortality of 10 or less per 1000 live birth by 2035 [37]. Further, the quality of neonatal resuscitation needs to be improved from that of the current state by improving health worker performance of neonatal resuscitation [7, 38]. Health workers need to be fully

Table 7 Logistic regression analysis on the level of association between different implementation strategies of the Helping Babies Breathe (HBB) Quality Improvement Cycle (QIC) and bag-and-mask skill retention

\begin{tabular}{lll}
\hline HBB QIC & Relative Risk & $95 \% \mathrm{Cl}$ \\
\hline Bag and mask skill check daily & 5.1 & $1.9-13.5$ \\
Preparation for resuscitation before & 2.4 & $1.0-5.6$ \\
every birth & & \\
Use of self-evaluation checklist & 3.8 & $1.4-9.7$ \\
Attendance of weekly review meeting & 2.6 & $1.0-7.4$ \\
\hline
\end{tabular}

competent in neonatal resuscitation skills, and further need to be able to retain this competency such that knowledge is translated into clinical practice to reduce the burden of intrapartum-related death and morbidity. Nepal's current national strategy to provide skilled attendance at birth through neonatal resuscitation training programs is not adequate to sustain clinical competency of neonatal resuscitation as shown by several previous studies [38-41]. A combination of strategies should be considered for implementation in health facilities across the country.

\section{Conclusion}

To our knowledge this is the first study done to evaluate the impact of a multi-faceted implementation strategy of $\mathrm{HBB}$ on the retention of neonatal resuscitation skills in low-income settings. We demonstrate that health workers, who conduct bag-and-mask skill checks daily, make preparation for resuscitation at every birth, use self-evaluation checklists after each delivery, and attend weekly review meetings have a higher likelihood for retention of neonatal resuscitation skills, even at 6 months post-training. Since, the HBB-QIC was implemented in a setting with a high number of deliveries, with a large team of nursing staff in each delivery unit, the implementation strategy such as weekly review meetings can be suitable in similar settings, but may not be suitable in primary care settings where there are fewer nursing staff. The other effective implementation strategies of daily bag-and-mask skill checks, preparation for resuscitation at every birth, and use of self-evaluation checklists can be applied in primary care settings to improve retention of resuscitation skills. However, the combination of different strategies needs to be evaluated in primary care settings, the point of access for delivery services among most women in rural areas. The innovative aspect of HBB-QIC is the multifaceted implementation strategy approach. Future research should focus on further determining and refining those components that improve retention of neonatal resuscitation skills and clinical performance.

\section{Abbreviations}

HBB: Helping Babies Breathe; QIC: Quality Improvement Cycle; MNSC: Maternal Newborn Service Center; OSCE: Objective structured clinical evaluation; IQR: Inter quartile range; SD: Standard deviation; RR: Relative risk

\footnotetext{
Acknowledgement

Laerdal Foundation for Acute Medicine (Stavanger, Norway) provided financial support this study as part of the larger study to evaluate the impact of a simplified neonatal resuscitation protocol on birth outcomes and health workers performance. Part of this study was supported through a grant from the Swedish Society of Medicine. MM, JW, UE and VN were supported through a grant from Uppsala University (Uppsala, Sweden). The study group would like to thank Professor Gehanath Baral, Dr. Sheela Verma and Dr. Dhan Raj Aryal who reviewed and provided input for the design and data collection components of the study. The group is very grateful to the health workers who participated in the study.
} 


\section{Funding}

Funding for the study was provided through grants from the Swedish Society of Medicine, Sweden and Laerdal Foundation for Acute Medicine (Stavanger, Norway). The views expressed in the publication is that of the authors and do not reflect the funding agency.

\section{Availability of data and materials}

The datasets used and analysed during the current study are available from the principal investigator with a reasonable request.

\section{Authors' contributions}

AK, MM, JW and UE conceptualized and designed the study. AK was the principal applicant for funding. AK was responsible for the data collection and data management. AK and VN were responsible for the data analysis and drafting of the manuscript. MM, JW, RC, UE, and SP contributed to the development of the manuscript. All authors read and approved the final manuscript.

\section{Competing interests}

The authors declare that they have no competing interests.

\section{Consent for publication}

Not applicable

\section{Ethics approval and consent to participate.}

As indicated in the section "Study design", the ethical review board of Nepal Health Research Council approved this study with reference Reg. No. 37/2012 on 16 July 2012. All participants were provided with written informed consent as indicated in the "Participants" section.

\section{Publisher's note}

Springer Nature remains neutral with regard to jurisdictional claims in published maps and institutional affiliations.

\section{Author details}

'International Maternal and Child Health, Department of Women's and Children's Health, Uppsala University, Uppsala, Sweden. 'United Nation's Children's Fund (UNICEF), Nepal Country Officer, UN House, Lalitpur, Nepal. ${ }^{3}$ Latter-day Saint Charities, Salt Lake City, UT, USA. ${ }^{4}$ Global Health, Public Health Services, Karolinska Institute, Stockholm, Sweden. ${ }^{5}$ School of Public Health, Makerere University, Kamala, Uganda. ${ }^{6}$ Health Section, Programme Division, United Nations Children Fund (UNICEF), United Nations Plaza, New York, NY 10017, USA.

\section{Received: 12 October 2015 Accepted: 31 March 2017}

\section{Published online: 11 April 2017}

\section{References}

1. Lawn JE, Blencowe H, Oza S, You D, Lee AC, Waiswa P, Lalli M, Bhutta Z, Barros AJ, Christian P, Mathers C, Cousens SN, Lancet every newborn study group. Every newborn: progress, priorities, and potential beyond survival. Lancet. 2014;384(9938):189-205.

2. Lee AC, Kozuki N, Blencowe H, Vos T, Bahalim A, Darmstadt GL, Niermeyer S, Ellis $\mathrm{M}$, Robertson NJ, Cousens S, Lawn JE. Intrapartum-related neonatal encephalopathy incidence and impairment at regional and global levels for 2010 with trends from 1990. Pediatr Res. 2013;74(Suppl 1):50-72.

3. Lee AC, Cousens S, Wall SN, Niermeyer S, Darmstadt GL, Carlo WA, Keenan WJ, Bhutta ZA, Gill C, Lawn JE. Neonatal resuscitation and immediate newborn assessment and stimulation for the prevention of neonatal deaths: a systematic review, meta-analysis and Delphi estimation of mortality effect. BMC Public Health. 2011;11(Suppl 3):S12

4. Murila F, Obimbo MM, Musoke R. Assessment of knowledge on neonatal resuscitation amongst health care providers in Kenya. Pan Afr Med J. 2012; 11:78.

5. Harvey SA, Blandon YC, McCaw-Binns A, Sandino I, Urbina L, Rodriguez C, Gomez I, Ayabaca P, Djibrina S. Are skilled birth attendants really skilled? A measurement method, some disturbing results and a potential way forward. Bull World Health Organ. 2007;85(10):783-90.

6. UNICEF, Government of Nepal. Multiple indicator cluster survey (MICS). Kathmandu: UNICEF; 2015.
7. Lindback C, KC A, Wrammert J, Vitrakoti R, Ewald U, Malqvist M. Poor adherence to neonatal resuscitation quidelines exposed; an observational study using camera surveillance at a tertiary hospital in Nepal. BMC Pediatr 2014, 14:233.

8. Grimshaw JM, Shirran L, Thomas R, Mowatt G, Fraser C, Bero L, Grilli R, Harvey E, Oxman A, O'Brien MA. Changing provider behavior: an overview of systematic reviews of interventions. Med Care. 2001;39(8 Suppl 2):I12-45.

9. Singhal N, Lockyer J, Fidler H, Keenan W, Little G, Bucher S, Qadir M, Niermeyer $\mathrm{S}$. Helping babies breathe: global neonatal resuscitation program development and formative educational evaluation. Resuscitation. 2012; 83(1):90-6.

10. Kattwinkel J. Neonatal resuscitation guidelines for ILCOR and NRP: evaluating the evidence and developing a consensus. J Perinatol. 2008; 28(Suppl 3):S27-9.

11. Roehr CC, Hansmann G, Hoehn T, Buhrer C. The 2010 guidelines on neonatal resuscitation (AHA, ERC, ILCOR): similarities and differences-what progress has been made since 2005? Klin Padiatr. 2011:223(5):299-307.

12. Msemo G, Massawe A, Mmbando D, Rusibamayila N, Manji K, Kidanto HL, Mwizamuholya D, Ringia P, Ersdal HL, Perlman J. Newborn mortality and fresh stillbirth rates in Tanzania after helping babies breathe training. Pediatrics. 2013;131(2):e353-60.

13. Vossius C, Lotto E, Lyanga S, Mduma E, Msemo G, Perlman J, Ersdal HL. Cost-effectiveness of the "helping babies breathe" program in a missionary hospital in rural Tanzania. PLoS One. 2014;9(7):e102080.

14. Hoban R, Bucher S, Neuman I, Chen M, Tesfaye N, Spector JM. 'Helping babies breathe' training in sub-saharan Africa: educational impact and learner impressions. J Trop Pediatr. 2013;59(3):180-6.

15. Musafili A, Essen B, Baribwira C, Rukundo A, Persson LA. Evaluating helping babies breathe: training for healthcare workers at hospitals in Rwanda. Acta Paediatr. 2013;102(1):e34-8.

16. Ersdal HL, Vossius C, Bayo E, Mduma E, Perlman J, Lippert A, Soreide E. A one-day "helping babies breathe" course improves simulated performance but not clinical management of neonates. Resuscitation. 2013;84(10):1422-7.

17. Ministry of Health and Population, GoN. Annual Report "Smarika" 2068/69. Kathmandu: Paropakar Maternity and Women's Hospital; 2011.

18. Ashish KC, Malqvist M, Wrammert J, Verma S, Aryal DR, Clark R, Naresh PK, Vitrakoti R, Baral K, Ewald U. Implementing a simplified neonatal resuscitation protocol-helping babies breathe at birth (HBB) - at a tertiary level hospital in Nepal for an increased perinatal survival. BMC Pediatr. 2012; 12:159.

19. Barnard J, Meng XL. Applications of multiple imputation in medical studies: from AIDS to NHANES. Stat Methods Med Res. 1999:8(1):17-36.

20. Smith KK, Gilcreast D, Pierce K. Evaluation of staff's retention of ACLS and BLS skills. Resuscitation. 2008;78(1):59-65.

21. Hamilton R. Nurses' knowledge and skill retention following cardiopulmonary resuscitation training: a review of the literature. J Adv Nurs. 2005:51(3):288-97.

22. Skidmore $M B$, Urquhart $H$. Retention of skills in neonatal resuscitation. Paediatr Child Health. 2001:6(1):31-5.

23. Kaczorowski J, Levitt C, Hammond M, Outerbridge E, Grad R, Rothman A, Graves L. Retention of neonatal resuscitation skills and knowledge: a randomized controlled trial. Fam Med. 1998;30(10):705-11.

24. Carlo WA, Wright LL, Chomba E, McClure EM, Carlo ME, Bann CM, Collins M, Harris $\mathrm{H}$. Educational impact of the neonatal resuscitation program in lowrisk delivery centers in a developing country. J Pediatr. 2009;154(4):504-8. e505

25. Enweronu-Laryea C, Engmann C, Osafo A, Bose C. Evaluating the effectiveness of a strategy for teaching neonatal resuscitation in West Africa. Resuscitation. 2009;80(11):1308-11.

26. Bould MD, Hayter MA, Campbell DM, Chandra DB, Joo HS, Naik VN. Cognitive aid for neonatal resuscitation: a prospective single-blinded randomized controlled trial. $\mathrm{Br} J$ Anaesth. 2009:103(4):570-5.

27. Reeves S, Zwarenstein M, Goldman J, Barr H, Freeth D, Hammick M, Koppel I. Interprofessional education: effects on professional practice and health care outcomes. Cochrane Database Syst Rev. 2008;1:CD002213.

28. Jamtvedt G, Young JM, Kristoffersen DT, Thomson O'Brien MA, Oxman AD. Audit and feedback: effects on professional practice and health care outcomes. Cochrane Database Syst Rev. 2003;3:CD000259.

29. Jamtvedt G, Young JM, Kristoffersen DT, O'Brien MA, Oxman AD. Audit and feedback: effects on professional practice and health care outcomes. Cochrane Database Syst Rev. 2006;2:CD000259. 
30. Ivers N, Jamtvedt G, Flottorp S, Young JM, Odgaard-Jensen J, French SD, O'Brien MA, Johansen M, Grimshaw J, Oxman AD. Audit and feedback: effects on professional practice and healthcare outcomes. Cochrane Database Syst Rev. 2012;6:CD000259.

31. Peters DH, SE S, Siadat B, Janovsky K, Vujicic M. Improving health service delivery in developing countries : from evidence to action. Washington: World Bank; 2009.

32. Forsetlund $\mathrm{L}$, Bjorndal $\mathrm{A}$, Rashidian $\mathrm{A}$, Jamtvedt $\mathrm{G}$, O'Brien MA, Wolf $\mathrm{F}$, Davis D, Odgaard-Jensen J, Oxman AD. Continuing education meetings and workshops: effects on professional practice and health care outcomes. Cochrane Database Syst Rev. 2009;2:CD003030

33. Pageler NM, Longhurst CA, Wood M, Cornfield DN, Suermondt J, Sharek PJ, Franzon D. Use of electronic medical record-enhanced checklist and electronic dashboard to decrease CLABSIs. Pediatrics. 2014;133(3):e738-46.

34. Davis DA, Taylor-Vaisey A. Translating guidelines into practice. A systematic review of theoretic concepts, practical experience and research evidence in the adoption of clinical practice guidelines. CMAJ. 1997;157(4):408-16.

35. Lion KC, Ebel BE, Rafton S, Zhou C, Hencz P, Mangione-Smith R. Evaluation of a quality improvement intervention to increase use of telephonic interpretation. Pediatrics. 2015;135(3):e709-16.

36. Ambroggio L, Thomson J, Murtagh Kurowski E, Courter J, Statile A, Graham C, Sheehan B, lyer S, Shah SS, White CM. Quality improvement methods increase appropriate antibiotic prescribing for childhood pneumonia. Pediatrics. 2013;131(5):e1623-31.

37. WHO, UNICEF. Every newborn: an action plan to end preventable deaths. Geneva: World Health Organization; 2014

38. UNICEF, Ministry of Health and Population. Assessment of readiness, availability and quality of neonatal services in 12 hospitals of Nepal. Kathmandu: UNICEF; 2014.

39. Ministry of Health and Population, GoN. National Neonatal Health Strategy2004. Kathmandu: Family Health Division; 2004.

40. Ministry of Health \& Population. National in-service training strategy for skilled birth attendant. Kathmandu: Ministry of Health \& Population; 2007.

41. Dickson, KE, Simen-Kapeu A, Kinney MV, Huicho L, Vesel L, Lackritz E, de Graft Johnson J, Von Xylander S, Rafique N, Sylla M, Mwansambo C, Daelmans B, Lawn JE The Lancet Every Newborn Study Group. Every Newborn: health-systems bottlenecks and strategies to accelerate scale-up in countries. Lancet. 2014;384(9941):438-54.

\section{Submit your next manuscript to BioMed Central and we will help you at every step:}

- We accept pre-submission inquiries

- Our selector tool helps you to find the most relevant journal

- We provide round the clock customer support

- Convenient online submission

- Thorough peer review

- Inclusion in PubMed and all major indexing services

- Maximum visibility for your research

Submit your manuscript at www.biomedcentral.com/submit 